Check for updates

Cite this: RSC Adv., 2019, 9, 33563

Received 26th August 2019

Accepted 8th October 2019

DOI: 10.1039/c9ra06709d

rsc.li/rsc-advances

\title{
A palladium complex confined in a thiadiazole- functionalized porous conjugated polymer for the Suzuki-Miyaura coupling reaction
}

\author{
Yingjie Qian, Sang Yung Jeong, Sung-Hyeon Baeck, (D) Myung-Jong Jin \\ and Sang Eun Shim (D)*
}

Porous organic polymers (POPs) with well-distributed and tunable functional groups acting as ligands for specific reactions are promising supports for confining useful novel metals such as $\mathrm{Pd}, \mathrm{Au}$, and $\mathrm{Pd}$. Herein, a thiadiazole-containing POP has been successfully synthesized and used for immobilizing Pd species. Pd immobilized inside the micropores $(2.3 \mathrm{~nm})$ of the POP material is easily prepared owing to a large amount of the strong anchoring group, thiadiazole, which is intrinsically distributed in the asprepared POP. The rigid thiadiazole-containing polymer can stabilize the central metal rather than poisoning it. The as-prepared catalyst shows excellent catalytic activity in Suzuki-Miyaura coupling reactions under mild reaction conditions and low catalyst loading. Importantly, the intrinsically distributed thiadiazole ligands can stabilize the Pd moiety, preventing aggregation and leaching, and afford excellent catalytic lifetimes. Consequently, the catalyst can be reused 10 times without a significant loss of its catalytic activity.
\end{abstract}

\section{Introduction}

Transition metal-catalyzed Suzuki-Miyaura coupling reactions have been ubiquitously employed in organic chemistry because of their applications in the synthesis of pharmaceuticals, natural products, and polymers. ${ }^{1}$ In general, the majority of the coupling reactions in the case of pharmaceuticals $^{1 a}$ and natural products ${ }^{1 b}$ rely on the use of homogeneous $\mathrm{Pd}$ complexes, which require an extensive separation and recycling of toxic and expensive catalysts from products. Additionally, increasing environmental exposure with the emission of Pd from organic transformations requires an effective control of the metal contamination of desired product and eco-system. ${ }^{2}$ Therefore, many approaches have been presented in previous studies focusing on the development of new strategies and supports to adequately immobilize the catalytic species. ${ }^{3}$ However, the application of heterogenized catalytic systems has been limited by leaching and deactivation over time due to the insufficient affinity between the central metal and support. Thus, it is essential to introduce substantial anchoring groups into a heterogeneous catalyst to enhance the long-term stability of the catalyst.

In the past few decades, an impressive number of solid supports, including silica matrices, graphene, metalorganic frameworks, metal oxides, and porous organic

Department of Chemistry \& Chemical Engineering, Inha University, 100 Inha-ro, Nam-gu, Incheon 22212, South Korea.E-mail: seshim@inha.ac.kr polymers (POPs) have been developed. ${ }^{4}$ Among them, POPbased heterogeneous catalysts have attracted significant interest owing to their potential applications in energy and gas storage. ${ }^{5}$ Recently, these have also emerged as promising materials for anchoring Pd. ${ }^{6}$ Because of their controllable intrinsic functional groups and nanoporous structures with high stability in organic solvents, POPs are suitable supports in catalysis. Wang et al. reported an efficient heterogeneous catalytic system by anchoring Pd moiety onto imine-based conjugated POPs and demonstrated the possibility of POP applicability in Suzuki-Miyaura coupling reaction. ${ }^{6}$ Significantly high turnover frequency value was achieved by Zhu et al. using imine-tagged POP. ${ }^{7}$ Both representative POP-based immobilized catalysts exhibited excellent catalytic activities in coupling reactions. However, the limited reuse of heterogeneous catalysts still limits the practical use of POP-based heterogeneous catalysts in industrial sector.

Prior studies suggest that introducing foreign elements $(\mathrm{N}, \mathrm{O}, \mathrm{S})$ to the POP is crucial for enhancing the recycling performance. ${ }^{8}$ Among the electronegative foreign elements, $\mathrm{S}$, which is a widely known Pd poison owing to the strong interaction between $\mathrm{S}$ and $\mathrm{Pd}$, is of considerable interest. However, only a few studies have examined the coupling transformation of sulfur-containing heterogeneous catalysts. ${ }^{8 \boldsymbol{b}, 9}$ Zhang et al. reported that the encapsulation of metal inside the cavities of POP was crucial and beneficial in the Suzuki-Miyaura coupling reactions in the presence of strong anchoring thiol groups. ${ }^{8 b}$ Additionally, a rigid, 
(a)

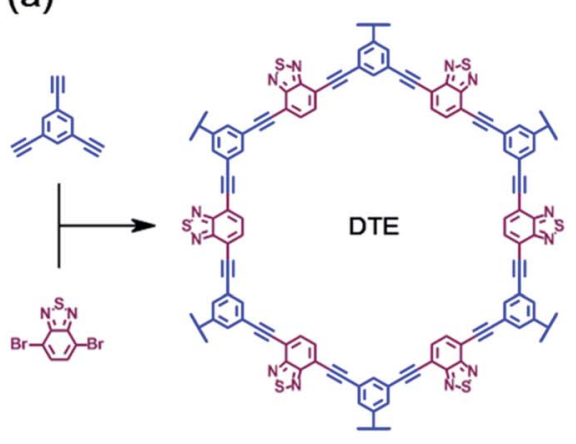

(b)

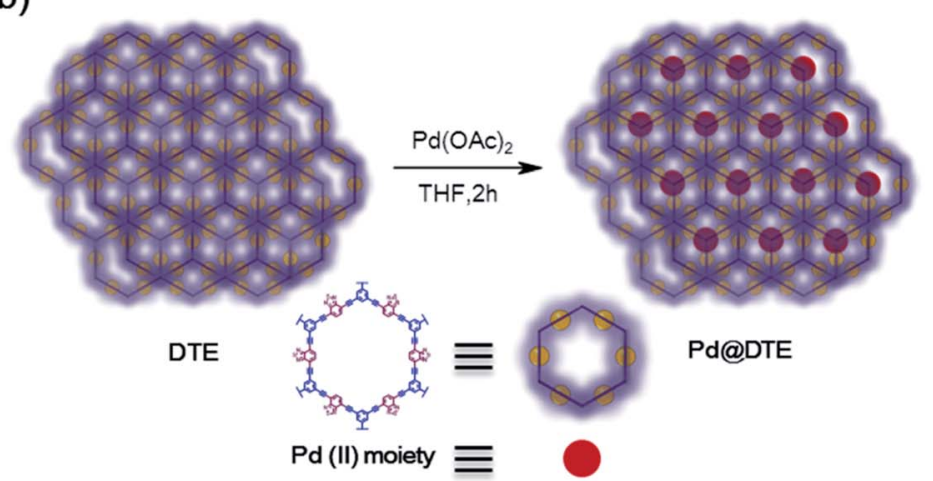

Scheme 1 (a) Synthesis of fully conjugated DTE and (b) schematic representation of the synthesis of DTE supported PdaDTE.

dangling thiol ligand, where the thiol groups are far apart to entirely bond the Pd species, was developed and it exhibited excellent reusability owing to the rigid framework of the sulfur-containing ligand. ${ }^{g}{ }^{5}$ Inspired by the previous investigations, it was envisioned that the presence of a rigid sulfur-containing anchoring groups that are evenly distributed inside the POP could facilitate the binding of the metal and ligand, thus enhancing the long-term stability of the catalyst. Herein, the synthesis of a thiadiazole-containing POP and its application in Suzuki-Miyaura coupling reactions is described. The synthesized catalyst exhibits the highest catalytic activity in comparison with the previously reported sulfur-containing heterogeneous catalysts. The catalyst also shows high stability over multiple catalytic cycles and easy recyclability.

\section{Experimental}

\section{Materials and characterization}

All chemicals were commercially available and used as-received without any further purification. 4,7-dibromobenzo[c][1,2,5] thiadiazole and tetrakis(triphenylphosphine)-palladium(0) were purchased from TCI. 1,3,5-Triethynylbenzene, copper(I)iodide (CUI), triethylamine (TEA), and all other chemicals were obtained from Sigma Aldrich. X-ray photoelectron spectroscopy (XPS) data were collected by using an ESCA 2000 photoelectron spectrometer (Thermo Scientific) with monochromated $\mathrm{Al} \mathrm{K} \alpha$ radiation $(h \nu=1486.6 \mathrm{eV})$. Fourier transform-infrared vacuum spectrometer (FT-IR) spectra were obtained by employing a Bruker VERTEX $80 \mathrm{~V}$. Solidstate NMR spectra were collected using a Bruker Digital AVANCE III (400 MHz) spectrometer. Field-emission transmission electron microscopy (FE-TEM) images were obtained by employing a JEOL JEM-2100F microscope. The Pd content was obtained through inductively coupled plasmaatomic emission spectroscopy (ICP-AES) using OPTIMA 4300 DV (Perkin-Elmer). The specific surface area and pore size distribution of the catalyst were investigated by measuring the nitrogen adsorption and desorption isotherms at $77 \mathrm{~K}$ on a BELSORP-MAX system (BEL, Japan). GC/GC-MS analyses were performed using an Agilent 6890N GC instrument coupled to an Agilent 5975 Network Mass Selective Detector.
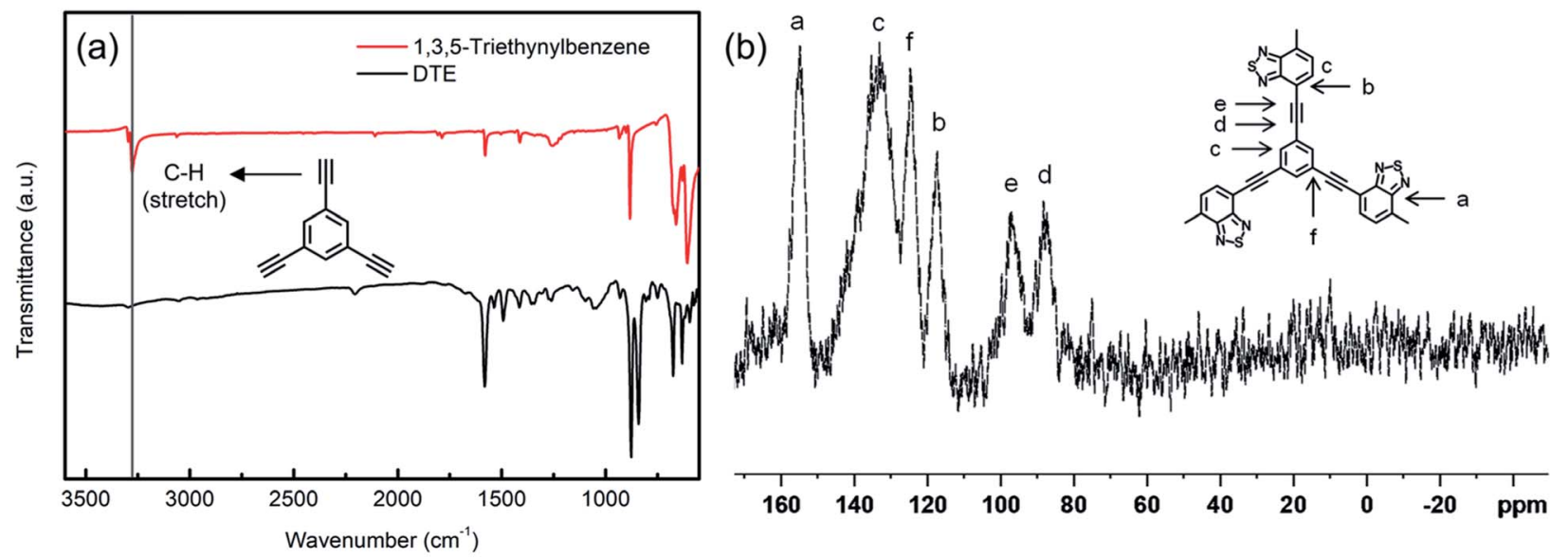

Fig. 1 (a) FT-IR spectra of 1,3,5-triethylnylbenzene and DTE and (b) solid-state ${ }^{13} \mathrm{C}$ NMR spectrum of DTE. 

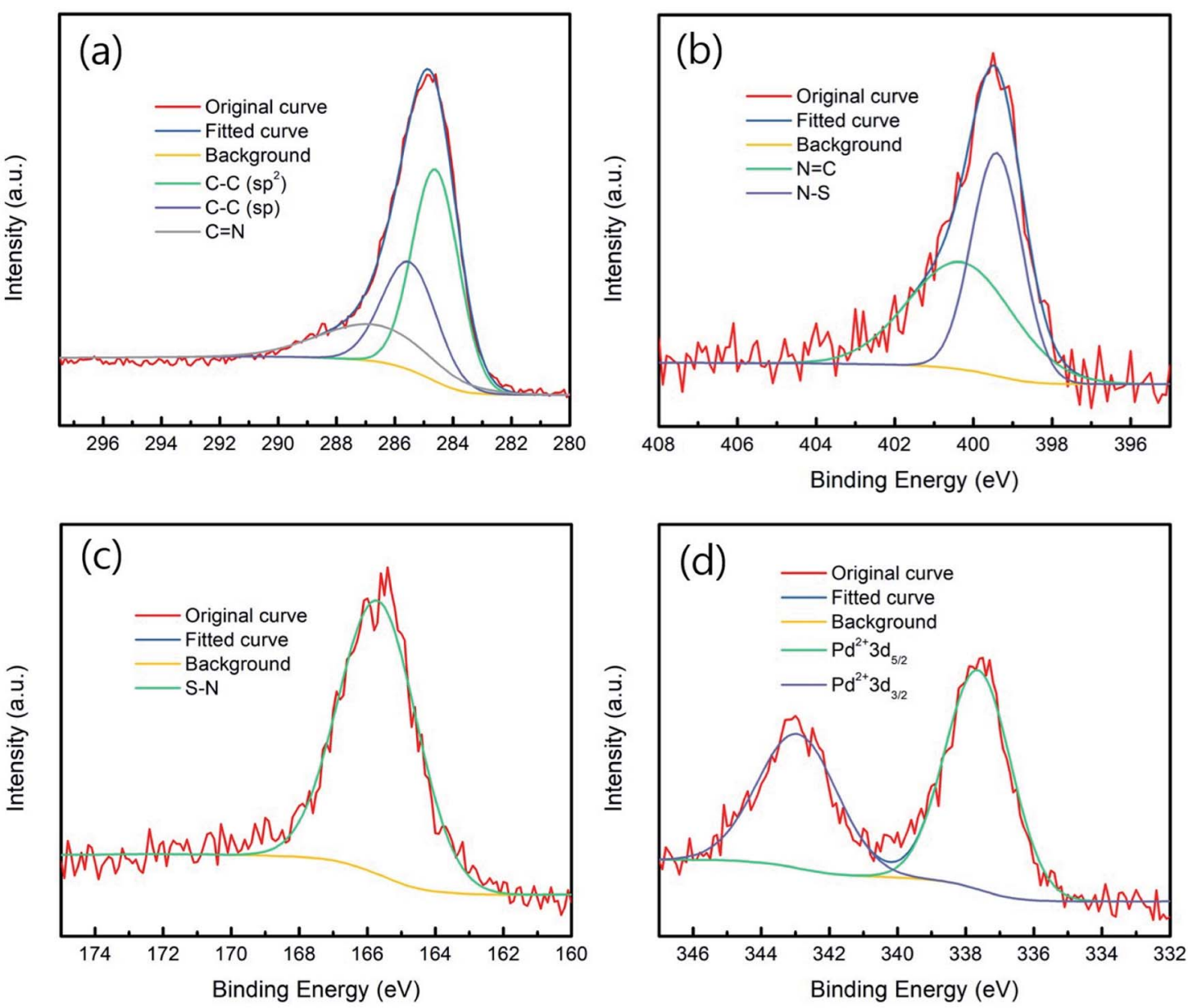

Fig. 2 (a) $C$ 1s. (b) $N$ 1s. (c) S 2p. (d) Pd 3d XPS spectra of PdaDTE.

\section{Synthesis of DTE}

4,7-Dibromobenzo[c] $[1,2,5]$ thiadiazole $(0.75 \mathrm{mmol})$ and 1,3,5-triethynylbenzene $(0.5 \mathrm{mmol})$ were dissolved in the mixture of anhydrous DMF/TEA $(15 \mathrm{ml} / 15 \mathrm{ml})$, named A, followed by purging for $10 \mathrm{~min}$. CuI $(0.04 \mathrm{mmol})$ and $\mathrm{Pd}$ catalyst $(0.025 \mathrm{mmol})$ were dissolved in TEA $(5 \mathrm{ml})$ and anhydrous DMF $(5 \mathrm{ml})$, respectively. After purging all solutions for $10 \mathrm{~min}$, the Pd and CuI solutions were simultaneously poured into solution A. The polymer DTE was synthesized at $120{ }^{\circ} \mathrm{C}$ for two days under an inert atmosphere. After the completion of the reaction, the generated polymer was purified by filtration, and washed with copious amounts of THF, acetone, DMF, and MC. The obtained DTE was dried at $75{ }^{\circ} \mathrm{C}$ under vacuum.

\section{Preparation of Pd-anchored DTE (Pd@DTE)}

DTE $(100 \mathrm{mg})$ was dispersed in anhydrous THF $(30 \mathrm{ml})$ and the mixture was ultrasonicated for $30 \mathrm{~min}$. After sonication, $\mathrm{Pd}(\mathrm{II})$ acetate $(5 \mathrm{mg}, 0.022 \mathrm{mmol})$ was added to the mixture, followed by stirring at $75^{\circ} \mathrm{C}$ for $2 \mathrm{~h}$. After the anchoring, the mixture was cooled down to room temperature and filtered, and thereafter washed with copious amounts of THF, MC, and diethyl ether. Pd@DTE was dried at $75{ }^{\circ} \mathrm{C}$ under vacuum. The Pd content of $0.18 \mathrm{mmol} \mathrm{g}^{-1}$ was measured by ICP-AES analysis.

\section{General procedure for the Suzuki coupling reaction}

Aryl bromide or chloride $(0.4 \mathrm{mmol})$, aryl boronic acid (0.48 $\mathrm{mmol})$, TBAB $(0.2 \mathrm{mmol}), \mathrm{K}_{2} \mathrm{CO}_{3}(0.8 \mathrm{mmol})$, and Pd@DTE (2.0 mg, $0.1 \mathrm{~mol} \%$ ) were mixed in a specific amount of $\mathrm{H}_{2} \mathrm{O}$ / EtOH. The mixture was stirred at a specific temperature under air. The reaction was monitored by GC. In the recycling experiment, after the reaction was completed, the catalyst was recovered simply by filtration. The separated catalyst was washed with water, ethanol, and diethyl ether. Thereafter, it was used in the next run under the same reaction conditions. The catalytic performance was confirmed by GC in the presence of $n$ dodecane as an internal standard.

\section{Results and discussion}

As shown in Scheme 1, the catalyst can be prepared in two steps. First, the fabrication of polymer DTE is achieved using the Sonogashira coupling reaction. Pd@DTE is obtained by immobilizing $\operatorname{Pd}(\mathrm{II})$ acetate to DTE. The structural 


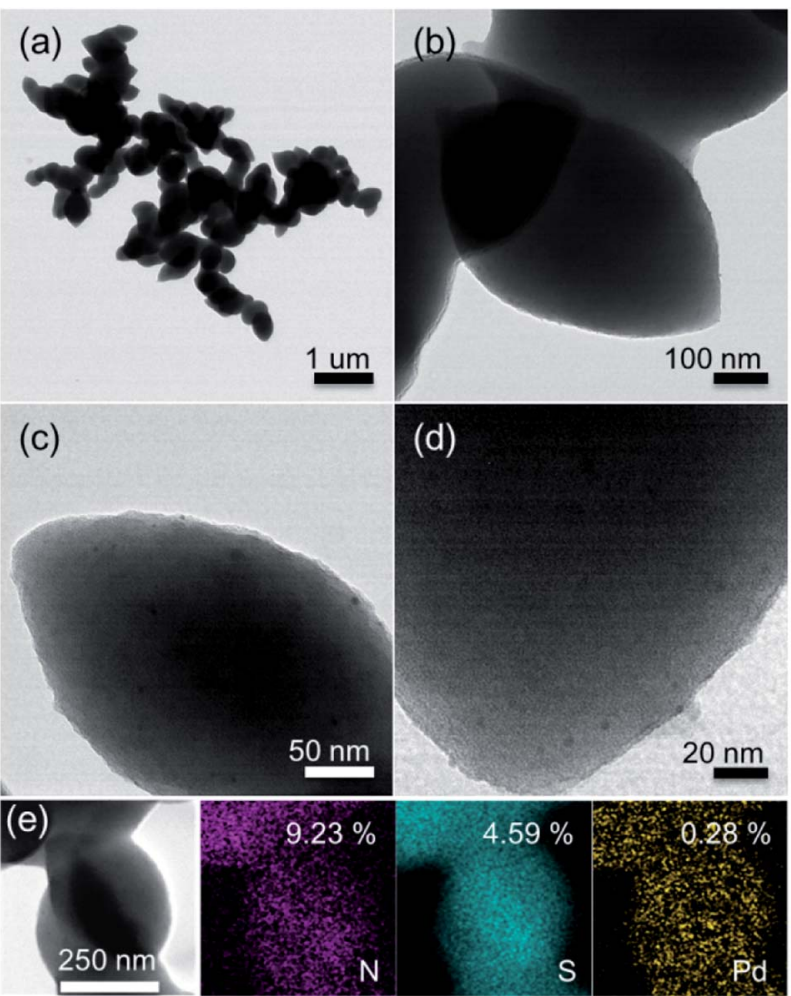

Fig. 3 Characterizations of Pd@DTE: ( $a$ and b) low-magnification TEM images. (c and d) High-magnification TEM images. (e) Energy dispersive $\mathrm{X}$-ray spectroscopy (EDS) mapping of composition element, $M, S$, and $\mathrm{Pd}$, respectively.

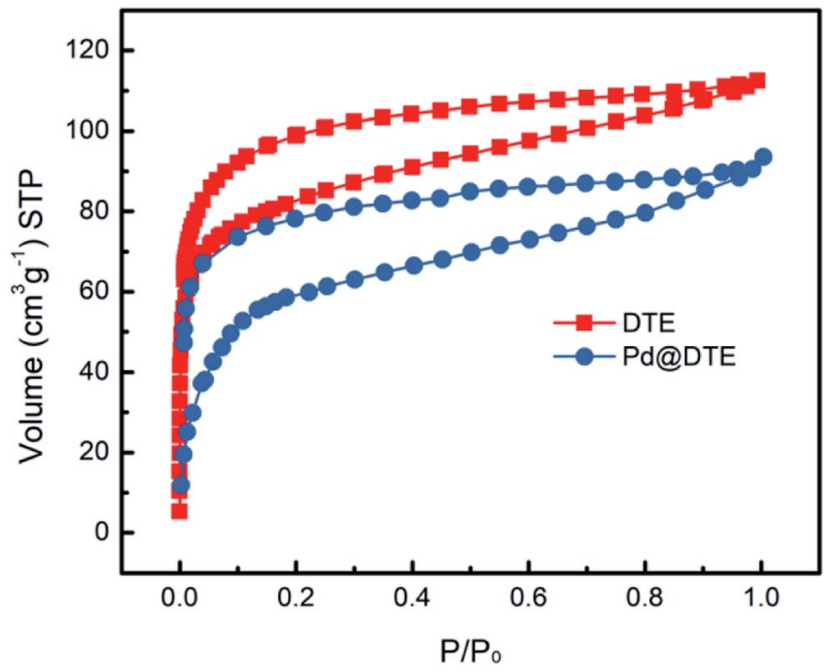

Fig. 4 Nitrogen adsorption-desorption isotherms of DTE and PdaDTE.

characteristics of the as-synthesized DTE were determined by FT-IR and solid-state ${ }^{13} \mathrm{C}$ NMR (Fig. 1). The disappearance of the $\mathrm{C}-\mathrm{H}$ stretch band at $3278 \mathrm{~cm}^{-1}$ and corresponding peaks from ${ }^{13} \mathrm{C}$ NMR spectrum illustrate the successful synthesis of DTE from building units. ${ }^{10}$ Next, XPS was employed to obtain the chemical states of elements and elemental composition of the samples. The $\mathrm{C}$ spectrum is deconvoluted and split into three peaks at 284.6, 285.7, and $287 \mathrm{eV}$, which are attributed to $\mathrm{sp}^{2}$, sp, and imine $\mathrm{C}(\mathrm{C}=\mathrm{N})$, respectively (Fig. 2a). The nitrogen spectrum of Pd@DTE shows the binding energies of 399.45 and $400.38 \mathrm{eV}$, corresponding to the imine $\mathrm{N}(\mathrm{N}=\mathrm{C})$ and $\mathrm{N}-\mathrm{S}$, respectively (Fig. 2b). Notably, the ratio of $\mathrm{N}=\mathrm{C}$ to $\mathrm{N}-\mathrm{S}$ is close to 1 , which implies that the intrinsic structure is maintained even after the anchoring of Pd moiety to DTE. S and Pd spectra are also deconvoluted and show three peaks centered at 165.8, 337.78, and $343.08 \mathrm{eV}$ which correspond to $\mathrm{S}-\mathrm{N}, \mathrm{Pd}^{2+}$ (Fig. 2c and d). ${ }^{11}$

The morphology of Pd@DTE was determined by TEM. Fig. 3a-d shows the images of fresh Pd@DTE at different magnifications. Fig. 3e illustrates the EDS mapping of the composition elements, nitrogen, sulfur, and palladium. Based on the EDS mapping results, DTE is uniformly anchored to Pd moiety. Moreover, the elemental composition of Pd@DTE is close to the theoretical values. Because of the large size of DTE, it is difficult to visualize the distribution of nano-sized Pd.

The nitrogen isotherm was collected at $77 \mathrm{~K}$ to illustrate the effect of Pd@DTE fabrication process (Fig. 4). The results for BET surface area $\left(S_{\mathrm{BET}}\right)$ and total pore volumes $\left(V_{\text {total }}\right)$ of DTE and Pd@DTE are summarized in Table 1. Both isotherms with type IV hysteresis show micro- and mesoporous characteristics. After the anchoring of Pd moieties, $S_{\mathrm{BET}}$ of catalyst 4 decreases from $309 \pm 7$ to $244 \pm 5 \mathrm{~m}^{2} \mathrm{~g}^{-1}$. As some of the micropores are filled with $\mathrm{Pd}$ moieties during the Pd treatment process, the decrease in micropores can be predicted. Meanwhile, it demonstrates that Pd species are successfully entered into the pores.

2-Bromoanisole was initially selected as a model starting material to optimize the reaction conditions (Table 2). First, the reaction was carried out with $0.1 \mathrm{~mol} \%$ of catalyst Pd@DTE and 2.0 eq. $\mathrm{K}_{2} \mathrm{CO}_{3}$ in different solvents at $50{ }^{\circ} \mathrm{C}$ (entries 1-6). Based on the solvent effect, EtOH was found to be the most effective organic solvent. EtOH/ $\mathrm{H}_{2} \mathrm{O}$ was used as the reaction media because this aqueous-organic media allowed the highest transformation performance in most cases. Furthermore, $\mathrm{K}_{2} \mathrm{CO}_{3}, \mathrm{KOH}, \mathrm{Na}_{2} \mathrm{CO}_{3}, \mathrm{KO}^{\mathrm{t}} \mathrm{Bu}$, and TEA were also tested and $\mathrm{K}_{2} \mathrm{CO}_{3}$ was determined to be the most effective base (entries 711).

To maximize the catalytic performance of Pd@DTE, the ratio of $\mathrm{H}_{2} \mathrm{O}$ to EtOH was varied. Fig. 5 shows the yield as a function of time for Suzuki-Miyaura coupling reaction of 2-bromoanisole with phenylboronic acid at different volume ratios of $\mathrm{H}_{2} \mathrm{O}$ to EtOH. Among the various combinations of the reaction media, the highest yield of $97.2 \%$ is obtained at $\mathrm{H}_{2} \mathrm{O}$ : EtOH of 2 . The difference in catalytic activity at different ratios of reaction media allows the modification and enhancement of the catalytic activity.

The Suzuki-Miyaura coupling reactions of aryl halides with aryl boronic acid were carried out under the optimized reaction conditions (Table 3). The sterically hindered 2,5-dibromotoluene and deactivated 4-bromophenol, 2-bromoanisole, and 4bromoanisole show good catalytic activities in $6 \mathrm{~h}$ (entries 1-5). 
Table 1 BET surface area $\left(S_{\mathrm{BET}}\right)$ and the total pore volumes $\left(V_{\text {total }}\right)$ from $\mathrm{N}_{2}$ adsorption-desorption isotherms for DTE and Pd@DTE

\begin{tabular}{|c|c|c|c|c|c|}
\hline Sample & $S_{\text {BET }}\left[\mathrm{m}^{2} \mathrm{~g}^{-1}\right]$ & $V_{\text {total }}\left[\mathrm{m}^{2} \mathrm{~g}^{-1}\right]$ & $V_{\text {meso }}\left[\mathrm{m}^{2} \mathrm{~g}^{-1}\right]$ & $V_{\text {micro }}\left[\mathrm{m}^{2} \mathrm{~g}^{-1}\right]$ & $\begin{array}{l}\text { Pore size } \\
{[\mathrm{nm}]}\end{array}$ \\
\hline DTE & $309 \pm 7$ & 0.174 & 0.094 & 0.08 & 2.3 \\
\hline Pd@DTE & $244 \pm 5$ & 0.141 & 0.075 & 0.066 & 2.2 \\
\hline
\end{tabular}

Table 2 Optimization for Suzuki-Miyaura coupling of 2-bromoanisole with phenylboronic acid ${ }^{a}$

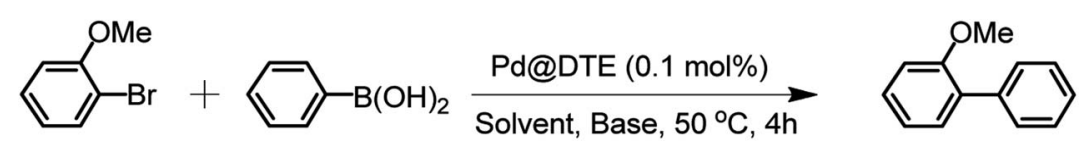

\begin{tabular}{|c|c|c|c|c|}
\hline Entry & Base & Catalyst (mol\%) & Solvent (total $2 \mathrm{ml}$ ) & Yield $^{b}(\%)$ \\
\hline 1 & $\mathrm{~K}_{2} \mathrm{CO}_{3}$ & 0.1 & $\mathrm{H}_{2} \mathrm{O}$ & 57.5 \\
\hline 2 & $\mathrm{~K}_{2} \mathrm{CO}_{3}$ & 0.1 & EtOH & 42.0 \\
\hline 4 & $\mathrm{~K}_{2} \mathrm{CO}_{3}$ & 0.1 & Toluene & 0.7 \\
\hline 5 & $\mathrm{~K}_{2} \mathrm{CO}_{3}$ & 0.1 & Propylene glycol & 20.4 \\
\hline 6 & $\mathrm{~K}_{2} \mathrm{CO}_{3}$ & 0.1 & $\mathrm{DMF}$ & 0.8 \\
\hline 9 & $\mathrm{KO}^{\mathrm{t}} \mathrm{Bu}$ & 0.1 & $\mathrm{EtOH} / \mathrm{H}_{2} \mathrm{O}(1: 1)$ & 58.1 \\
\hline 10 & $\mathrm{Na}_{2} \mathrm{CO}_{3}$ & 0.1 & $\mathrm{EtOH} / \mathrm{H}_{2} \mathrm{O}(1: 1)$ & 81.9 \\
\hline 11 & $\mathrm{NEt}_{3}$ & 0.1 & $\mathrm{EtOH} / \mathrm{H}_{2} \mathrm{O}(1: 1)$ & 37.2 \\
\hline
\end{tabular}

${ }^{a}$ Reaction conditions: aryl halide $(0.4 \mathrm{mmol})$, boronic acid $(0.48 \mathrm{mmol})$, Pd@DTE $(0.1 \mathrm{~mol} \%)$, base $(0.8 \mathrm{mmol})$, TBAB $(0.2 \mathrm{mmol})$, solvent $(2 \mathrm{ml})$, $50{ }^{\circ} \mathrm{C} .{ }^{b} \mathrm{GC}$ yield was determined using $n$-dodecane as an internal standard.

Activated compounds such as 4-suitable, 4-bromobenzene, 4bromobenzonitrile, and 2-bromonitrobenzene exhibit excellent performances (entries 6-9). The sterically hindered 3-bromo-4- methyl acetophenone shows excellent results because of the existence of an activating acetophenone moiety in the molecular structure (entry 10).<smiles>COc1ccccc1-c1ccccc1-c1ccccc1OB(O)c1ccccc1</smiles>
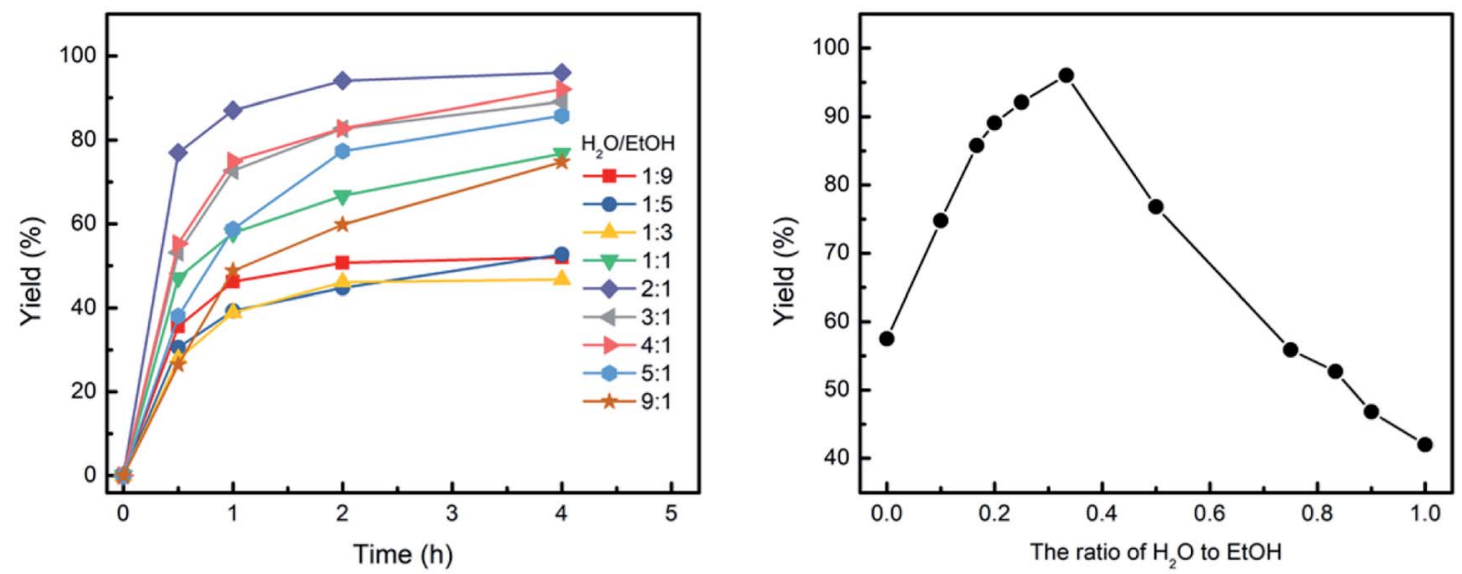

Fig. 5 The yield versus time for Suzuki-Miyaura coupling reaction of 2-bromoanisole with phenylboronic acid at different volume ratio of $\mathrm{H}_{2} \mathrm{O}$ and $\mathrm{EtOH}$ at $50^{\circ} \mathrm{C}$. 


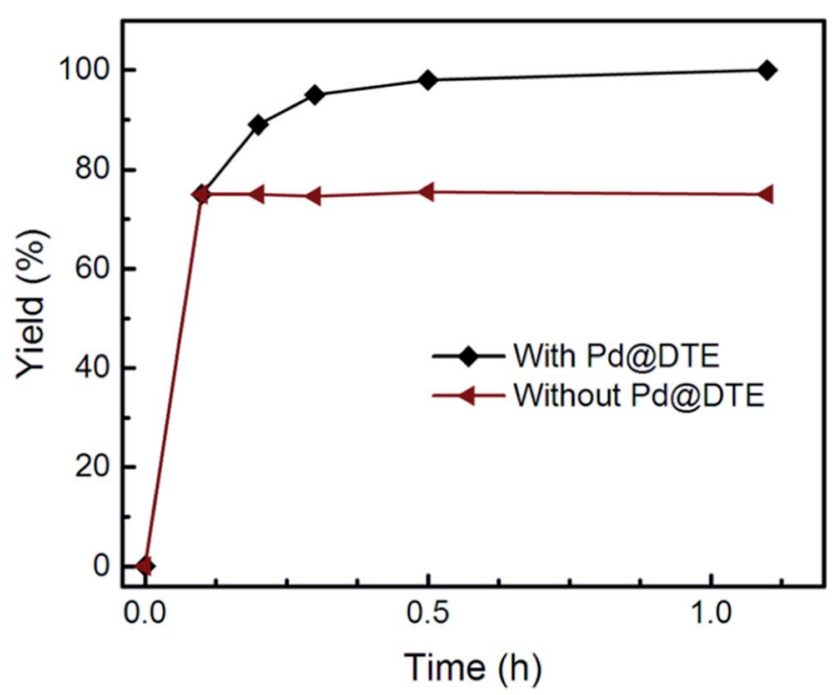

Fig. 6 Hot filtration experiments. Suzuki-Miyaura coupling reaction of 2 -bromoanisole with phenylboronic acid was carried out at $100{ }^{\circ} \mathrm{C}$.
Encouraged by the results of these coupling reactions, some hetero halides and severely sterically hindered aryl halides were also investigated (Table 4). Coupling of the heteroaryl structure is more difficult than aryl halides owing to presence of elements such as nitrogen or sulfur, which deactivate the catalyst. Therefore, the investigation of the performance of heteroaryls is also important. 2-Bromothiophene shows excellent yield in $20 \mathrm{~h}$ (entry 1). Additionally, 2-bromo-3-methylpyridine, 2-bromopyridine, and 3bromopyridine can couple in good to excellent yields at $50{ }^{\circ} \mathrm{C}$ in $20 \mathrm{~h}$ (entries $2-4$ ). The excellent catalytic activity of Pd@DTE is also supported by the coupling of 2,5-dibromopyridine, 2,5-dibromothiophene, 2-bromo-3-aminopyridine, and mesitylene bromide in good yields (entries 5-8).

The reusability of the catalyst is one of the most important features of an attractive heterogeneous catalyst. The recycling of Pd@DTE was examined in the Suzuki-Miyaura coupling of 4-iodobenzene with phenylboronic acid (Table 5) The catalytic system exhibits excellent reusability even

Table 3 Suzuki-Miyaura coupling of aryl halides with arylboronic acids ${ }^{a}$

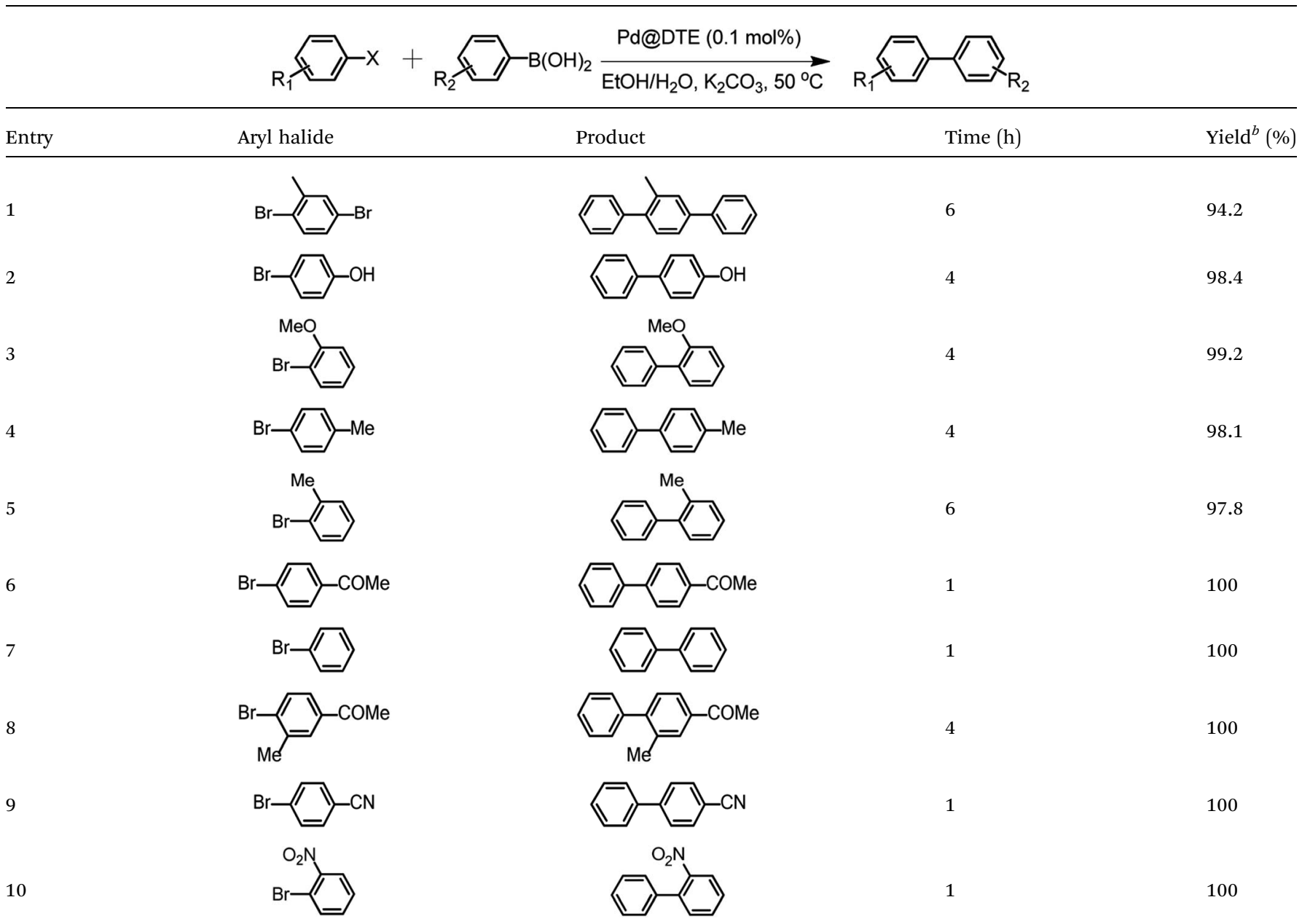
${ }^{a}$ Reaction conditions: aryl halide $(1 \mathrm{mmol})$, arylboronic acid $(1.5 \mathrm{mmol}), \mathrm{Pd} @ \mathrm{DTE}(0.1 \mathrm{~mol} \%), \mathrm{K}_{2} \mathrm{CO}_{3}(2.0 \mathrm{mmol}), \mathrm{TBAB}(0.5 \mathrm{mmol}), \mathrm{H}_{2} \mathrm{O}(2.0 \mathrm{ml})$,
EtOH $(2.0 \mathrm{ml}), 50{ }^{\circ} \mathrm{C} .{ }^{b} \mathrm{GC}$ yield was determined using $n$-dodecane as an internal standard. 
Table 4 Suzuki-Miyaura coupling of aryl halides with arylboronic acids ${ }^{a}$

Entry

${ }^{a}$ Reaction conditions: aryl halide $(1 \mathrm{mmol})$, arylboronic acid $(1.5 \mathrm{mmol}), \mathrm{Pd} @ D T E(0.1 \mathrm{~mol} \%), \mathrm{K}_{2} \mathrm{CO}_{3}(2.0 \mathrm{mmol}), \mathrm{TBAB}(0.5 \mathrm{mmol}), \mathrm{H}_{2} \mathrm{O}(2.0 \mathrm{ml})$, EtOH $(2.0 \mathrm{ml}), 50{ }^{\circ} \mathrm{C} .{ }^{b} \mathrm{GC}$ yield was determined using $n$-dodecane as an internal standard. ${ }^{c}$ The reaction was carried out at $75{ }^{\circ} \mathrm{C}$.

Table 5 Reusability of Pd@DTE in the Suzuki-Miyaura coupling ${ }^{a}$

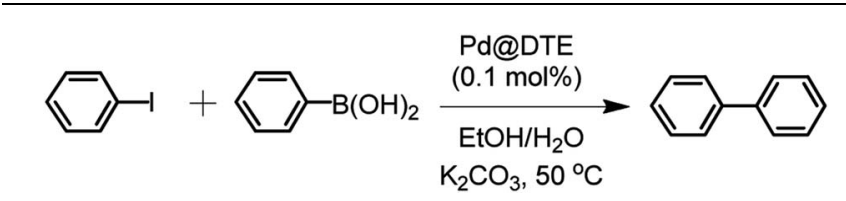

\begin{tabular}{lll}
\hline Entry & Time (h) & Yield $^{b}(\%)$ \\
\hline 1st batch & 1 & 100 \\
2nd batch & 1 & 100 \\
3rd batch & 1 & 99.1 \\
4th batch & 1 & 98.5 \\
5th batch & 1 & 99.2 \\
6th batch & 1 & 98.2 \\
7th batch & 1 & 98 \\
8th batch & 1 & 98.7 \\
9th batch & 1 & 98.4 \\
10th batch & 1 & 97.6
\end{tabular}

${ }^{a}$ Reaction conditions: aryl halide $(1 \mathrm{mmol})$, arylboronic acid $(1.5$ $\mathrm{mmol})$, Pd@DTE $(0.1 \mathrm{~mol} \%), \mathrm{K}_{2} \mathrm{CO}_{3}(2.0 \mathrm{mmol})$, TBAB $(0.5 \mathrm{mmol})$, $\mathrm{H}_{2} \mathrm{O}(2.0 \mathrm{ml})$, EtOH $(2.0 \mathrm{ml}), 50{ }^{\circ} \mathrm{C} .{ }^{b} \mathrm{GC}$ yield was determined using $n$-dodecane as an internal standard. after 10 cycles of reuse, which is an outstanding result among the sulfur-containing heterogeneous catalysts (Table 6). The high stability of the rigid sulfur-containing Pd@DTE catalysts in the reaction media is owing to the robust molecular structure with strong interaction between the Pd moiety and thiadiazole-containing framework.

To determine whether Pd@DTE is working in a heterogeneous manner, a hot-filtration test was carried out in the Suzuki-Miyaura coupling reaction of 2-bromoanisole with phenylboronic acid at $100{ }^{\circ} \mathrm{C}$ (Fig. 6). During the reaction, the heterogeneous catalyst was separated by filtration after $0.1 \mathrm{~h}$ (conversion 75\%); the reaction was carried out for a further $1 \mathrm{~h}$. The GC analysis showed that the supernatant has no catalytic activity. ICP-OES analysis of the supernatant confirms that $\mathrm{Pd}$ is absent in the solution. These results suggested that no leaching of Pd species takes place during the catalysis and demonstrated the effectiveness of the Pd@DTE in confining Pd moiety originated from the intrinsic features of DTE, including evenly distributed strong thiadiazole ligand and microporous structure. 
Table 6 Comparison of Pd@DTE with reported sulfur containing heterogeneous Pd catalysts

\begin{tabular}{|c|c|c|c|c|c|c|c|c|c|}
\hline Catalyst & Solvent & Temp. $\left({ }^{\circ} \mathrm{C}\right)$ & Loading (mol\%) & Time (h) & $\begin{array}{l}\text { Yield } \\
(\%)\end{array}$ & Substrate & $\begin{array}{l}\text { Number of cycles } \\
\text { \& Substrate }\end{array}$ & TOF & References \\
\hline PdNPs@COF & Water \& DMF & 50 & 0.1 & 3 & 82.9 & & 5 & 276 & $8 b$ \\
\hline ZrDMTD-Pd & Ethanol & 80 & 1 & 5 & 86 & & 8 & 17.2 & $9 b$ \\
\hline Pd-P/polythiophene & Water \& ethanol & RT & 1 & 24 & 87 & & $\mathrm{~N} / \mathrm{A}$ & 4 & $9 c$ \\
\hline
\end{tabular}

\section{Conclusion}

In summary, a rigid thiadiazole-containing porous polymer DTE is fabricated, followed by the immobilization of Pd moiety to DTE. First, the intrinsic sulfur ligand is retained during the preparation process of DTE at elevated temperature. Second, the Pd moiety is successfully anchored on DTE, which is confirmed by XPS and TEM data. According to the elemental analysis and TEM mapping results, we found that the elemental composition of as prepared DTE and Pd@DTE is very close to theoretical values. The catalytic activity was confirmed by carrying out the Suzuki-Miyaura coupling reactions of aryl and heteroaryl bromides. The catalyst showed excellent catalytic activity in coupling activated aryl bromides with phenylboronic acid in short reaction period. The highly sterically hindered mesitylene bromide and 2,5-dibromotoluene was coupled in 20 and $6 \mathrm{~h}$, respectively. Additionally, heteroaryl deactivated by foreign elements was also exhibited good to excellent catalytic activities in $20 \mathrm{~h}$. From the catalytic results the robustness structure of the DTE, and strong bond between rigid thiadiazole ligand and Pd moiety allow the excellent activity of catalyst and its reuse for at least 10 times without a significant loss of its catalytic activity.

\section{Conflicts of interest}

There are no conflicts to declare.

\section{Acknowledgements}

This work was supported by the Basic Science Research Program through the National Research Foundation of Korea (NRF), funded by the Ministry of Education (grant number: NRF-2015R1A4A1042434).

\section{Notes and references}

1 (a) C. E. Garrett and K. Prasad, Adv. Synth. Catal., 2004, 346, 889-900; (b) K. Konigsberger, G.-P. Chen, R. R. Wu, M. J. Girgis, K. Prasad, O. Repic and T. J. Blacklock, Org. Process Res. Dev., 2003, 7, 733-742; (c) Y. H. Xu, S. B. Jin, H. Xu, A. Nagai and D. L. Jiang, Chem. Soc. Rev., 2013, 42, 8012-8031.

2 J. Kiehorn, C. Melber, D. Keller and I. Mangelsdorf, Int. J. Hyg. Environ. Health, 2002, 205, 417-432.

3 (a) Y. X. Hou, X. M. Zhang, J. S. Sun, S. Lin, D. D. Qi, R. R. Hong, D. Q. Li, X. Xiao and J. Z. Jiang, Microporous Mesoporous Mater., 2015, 214, 108-114; (b) X. Y. Jiang, W. X. Zhao, W. Wang, F. Zhang, X. D. Zhuang, S. Han and X. L. Feng, Polym. Chem., 2015, 6, 6351-6357; (c) E. R. Rangel, E. M. Maya, F. Sanchez, J. G. D. L. Campa and M. Lglesias, Green Chem., 2015, 17, 466-473; (d) Q. Wen, T.-Y. Zhou, Q.-L. Zhao, J. Fu, Z. Ma and X. Zhao, Macromol. Rapid Commun., 2015, 36, 413-418; (e) M. Shunmughanathan, P. Puthiaraj and K. Pitchumani, ChemCatChem, 2015, 7, 666-673; (f) R. S. B. Goncalves, A. B. V. D. Oliveira, H. C. Sindra, B. S. Archanjo, M. E. Mendoza, L. S. A. Carneiro, C. D. Buarque and P. M. Esteves, ChemCatChem, 2016, 8, 743-750; $(g)$ X. M. Ren, S. N. Kong, Q. D. Shu and M. H. Shu, Chin. J. Chem., 2016, 34, 373-380; (h) Z. L. Du, Q.-Q. Dang and X.-M. Zhang, Ind. Eng. Chem. Res., 2017, 56, 4275-4280.

4 (a) D. K. Shen, L. Chen, J. P. Yang, R. Y. Zhang, Y. Wei, X. M. Li, W. Li, Z. K. Sun, H. W. Zhu, A. M. Abdullah, A. AlEnizi, A. A. Elzatahry, F. Zhang and D. Y. Zhao, ACS Appl. Mater. Interfaces, 2015, 7, 17450-17459; (b) L. Shang, T. Bian, B. H. Zhang, D. H. Zhang, L.-Z. Wu, C.-H. Tung, Y. D. Yin and T. R. Zhang, Angew. Chem., Int. Ed., 2014, 53, 250-254; (c) Q. H. Yang, Q. Xu and H.-L. Jiang, Chem. Soc. Rev., 2017, 46, 4774-4808; (d) W.-N. Wang, W.-J. An, B. Ramalingam, S. Mukherjee, D. M. Niedzwiedzki, 
S. Gangopadhyay and P. Biswas, J. Am. Chem. Soc., 2012, 134, 11276-11281; (e) Y.-B. Huang, Q. Wang, J. Liang, X. S. Wang and R. Cao, J. Am. Chem. Soc., 2016, 138, 10104-10107.

5 (a) P. Slavik, D. W. Kurka and D. K. Smith, Chem. Sci., 2018, 9, 8673-8681; (b) Y. Xie, T.-T. Wang, X.-H. Liu, K. Zou and W.-Q. Deng, Nat. Commun., 2013, 4, 1-7.

6 S.-Y. Ding, J. Gao, Q. Wang, Y. Zhang, W.-G. Song, C.-Y. Su and W. Wang, J. Am. Chem. Soc., 2011, 133, 19816-19822.

7 L.-P. Jing, J.-S. Sun, F. X. Sun, P. Chen and G. S. Zhu, Chem. Sci., 2018, 9, 3523-3530.

8 (a) Y. Z. Liao, Z. H. Cheng, W. W. Zuo, A. Thomas and C. F. J. Faul, ACS Appl. Mater. Interfaces, 2017, 9, 3839038400; (b) S. L. Lu, Y. M. Hu, S. Wan, R. McCaffrey, Y. H. Jin, H. W. Gu and W. Zhang, J. Am. Chem. Soc., 2017, 139, 17082-17088.
9 (a) R. L. Oliveira, W. He, R. J. M. K. Gebbink and K. P. D. Jong, Catal. Sci. Technol., 2015, 5, 1919-1928; (b) B. Gui, K.-K. Yee, Y.-L. Wong, S.-M. Yiu, M. Zeller, C. Wang and Z. T. Xu, Chem. Commun., 2015, 51, 6917-6920; (c) S.-E. Bae, K.-J. Kim, Y.-K. Hwang and S. Huh, ýJ. Colloid Interface Sci., 2015, 456, 93-99; (d) G. W. Yun, Z. Hassan, J. Y. Lee, J. H. Kim, N.-S. Lee, N. H. Kim, K. K. Baek, I. Hwang, C. G. Park and K. M. Kim, Angew. Chem., Int. Ed., 2014, 53, 6414-6418.

10 W. Huang, B. C. Ma, D. Wang, Z. J. Wang, R. Li, L. Wang, K. Landfester and K. A. I. Zhang, J. Mater. Chem. A, 2017, 5, 3792-3797.

11 Q. S. Zhao, Y. Z. Zhu, Z. Sun, Y. Li, G. L. Zhang, F. B. Zhang and X. B. Fan, J. Mater. Chem. A, 2015, 3, 2609-2616. 